\title{
Sepsis-Update: Definition und Epidemiologie
}

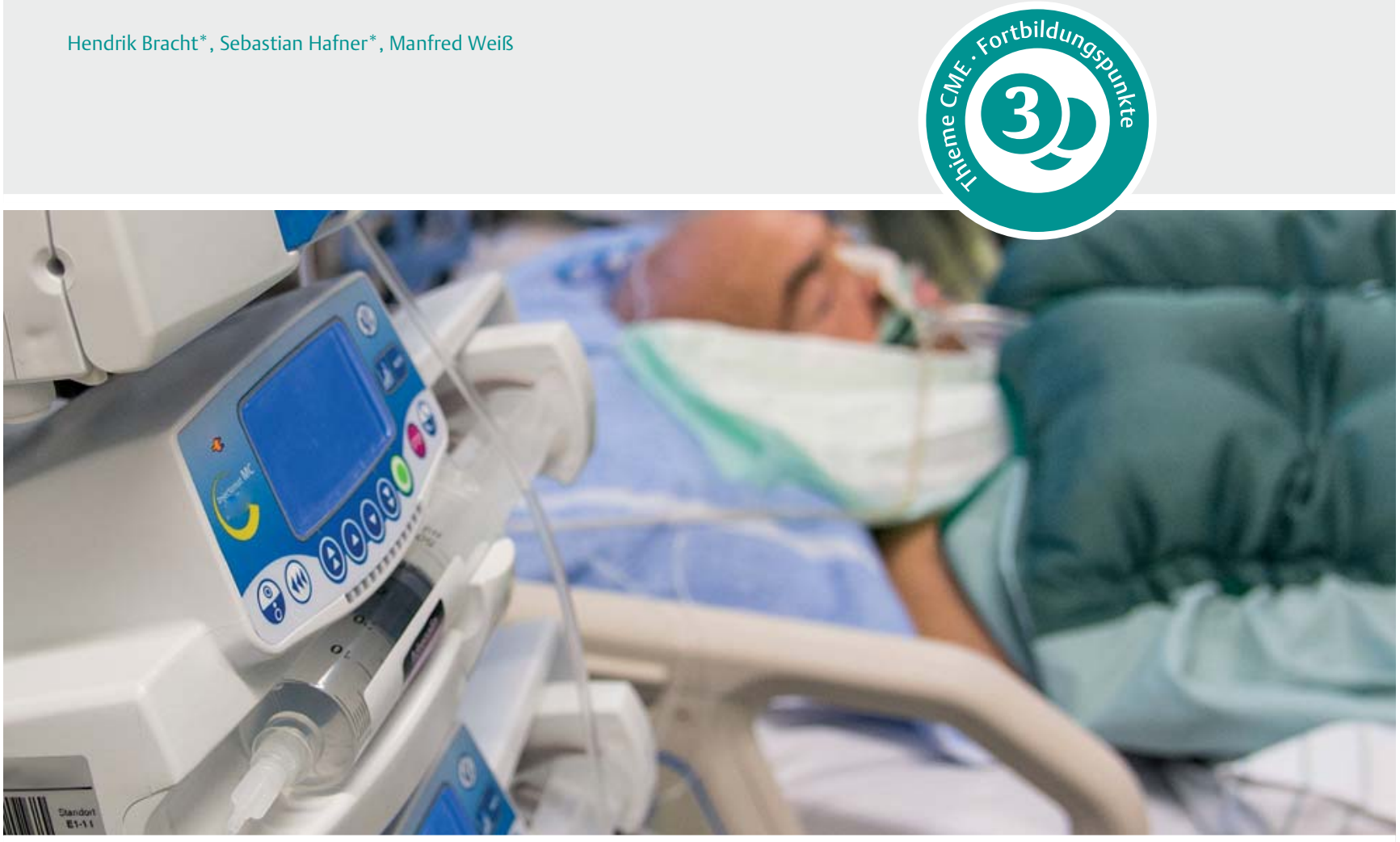

Quelle: KH Krauskopf.

Sepsis ist eine häufige Erkrankung auf den Intensivstationen weltweit, die mit einer relevanten Morbidität und Mortalität einhergeht. Bis heute gibt es keine spezifische Therapie der Sepsis - allein die frühe Diagnosestellung und schnellstmögliche therapeutische Intervention können das Outcome der Patienten verbessern. Daher ist eine Definition des Krankheitsbildes der Sepsis mit hoher diagnostischer Sensitivität und Spezifität unabdingbar.

\section{Einführung}

Obwohl seit Jahrzehnten massive Forschungsbemühungen unternommen werden, existiert bis dato für die Sepsis keine spezifische Therapie. Entscheidend in der Behandlung der Sepsis sind die frühe Sanierung des Infektfokus, die schnellstmögliche Gabe einer breiten, kalkulierten Antibiose sowie die Stabilisierung der hämodynamischen Situation. Hohe diagnostische Sensitivität und Spezifität der Sepsisdefinition sind deshalb extrem wichtig für den rechtzeitigen Beginn der Therapie und somit die Prognose der betroffenen Patienten.

* Hendrik Bracht und Sebastian Hafner haben gleichermaßen zur Arbeit beigetragen.

\section{Definition der Sepsis}

\section{Sepsis-1/Sepsis-2}

Bereits 1991 wurden in einer Konsensuskonferenz allgemein gültige Kriterien zur Definition der Sepsis festgelegt [1]. Diese Definition beruhte auf dem Konzept des systemischen inflammatorischen Response-Syndroms (SIRS). Je nach Schweregrad der Erkrankung wurde unterschieden zwischen

- Sepsis,

- schwerer Sepsis (mit Organdysfunktion) und

- septischem Schock (Sepsis und flüssigkeitsrefraktäre Hypotonie mit konsekutiver Katecholaminpflichtigkeit). 
Ausschlaggebend waren das Vorliegen von mind. 2 der 4 sog. SIRS-Kriterien sowie eine vermutete oder nachgewiesene Infektion ( $\triangleright$ Abb. 1). Diese Kriterien umfassten - Hypo- $\left(\leq 36^{\circ} \mathrm{C}\right)$ oder Hyperthermie $\left(\geq 38^{\circ} \mathrm{C}\right)$,

- Tachykardie ( $\geq 90 / \mathrm{min})$,

- Tachypnoe ( $\geq 20 / \mathrm{min})$ sowie

- Leukozytose $\geq 12000 / \mu \mathrm{l}$ sowie $\mathrm{PaCO}_{2}<32 \mathrm{mmHg}$ oder Leukopenie $\leq 4000 / \mu l$ und/oder Linksverschiebung $>10 \%$.

Die 1991 festgelegte Definition wurde durch eine erneute Konsensuskonferenz unter Mitwirkung von europäischen und amerikanischen Fachgesellschaften im Jahre 2001 überarbeitet [2]. Es wurden nun mehr als die bisher verwendeten 4 klinischen Kriterien zur Diagnosestellung herangezogen und für 8 unterschiedliche Organsysteme Dysfunktionsgrenzwerte festgelegt.

\section{Kritik an Sepsis-1/Sepsis-2}

Die beiden Definitionen Sepsis-1 und Sepsis-2 beruhten auf der Annahme, dass die überschießende generalisierte Entzündungsreaktion verantwortlich ist für die Pathogenese der Sepsis. Die Definition der Sepsis als Reaktion auf eine Infektion, die mit mind. 2 von 4 SIRS-Kriterien einhergeht, wurde in der Vergangenheit zunehmend kritisch hinterfragt. Ein Kritikpunkt war, dass die zwar sehr hohe Sensitivität in der Diagnosestellung eines SIRS mit einer nur geringen Spezifität für die Erfassung einer Sepsis einherging. Auf der anderen Seite gab es Patienten mit lebensbedrohlicher Infektion, die zwar klinisch eindeutig als septisch diagnostiziert werden konnten - sie wurden jedoch von der Sepsis-1- oder Sepsis-2-Definition nicht als „septisch“ erfasst.

\section{Sepsis-3}

Ein verändertes Verständnis der Pathophysiologie der Sepsis führte dazu, dass im Jahre 2014 eine weitere Konsensuskonferenz ins Leben gerufen wurde: Durch eine systematische Literaturrecherche und einen Delphi-Prozess wurde eine neue Sepsisdefinition erstellt. Zu dieser Änderung der Sepsisdefinitionen führten [3 - 5]

- zum einen das umfassendere pathophysiologische Verständnis,

- die Tatsache, dass hospitalisierte Patienten die SIRSKriterien auch ohne vorliegende Infektion erfüllen können, sowie

- der Umstand, dass 1 von 8 Patienten mit Infektion und neuer Organdysfunktion nicht mind. 2 SIRS-Kriterien aufweist.

Die nun vorliegende Definition ist die erste empirische, evidenz- und datenbasierte Beschreibung der Sepsis.

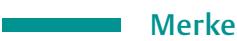

Als Sepsis wird seit 2016 eine lebensbedrohliche Organdysfunktion definiert, die durch eine dysregulierte Immunantwort der Patienten auf eine Infektion verursacht wird [5].

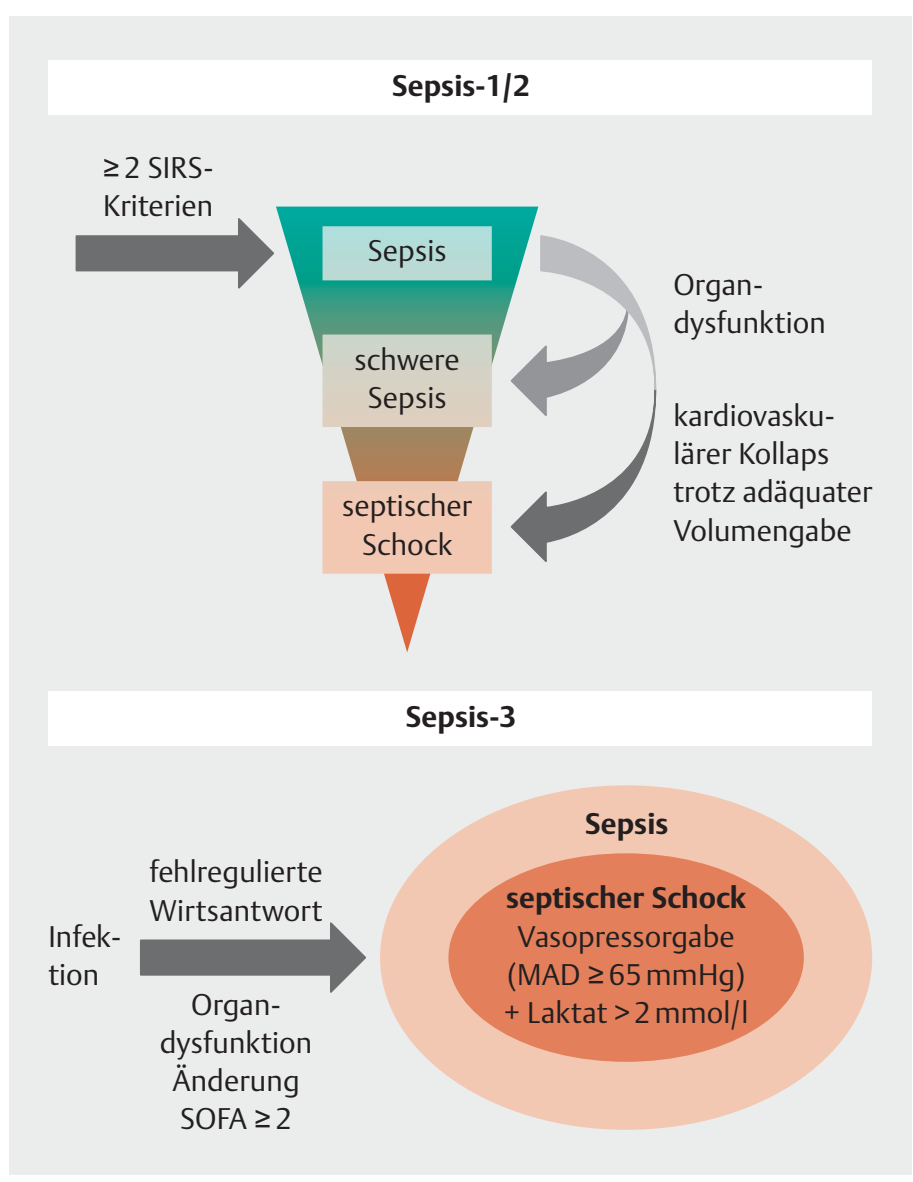

Abb. 1 Schematische Darstellung der Sepsisdefinitionen Sepsis-1/2 [1, 2] und Sepsis-3 [5]. SIRS: Systemic Inflammatory Response Syndrome, SOFA-Score: Sequential Organ Failure Assessment Score, MAD: mittlerer arterieller Druck.

SIRS wird in der Sepsis-3-Definition nicht mehr verwendet, ebenso der Begriff schwere Sepsis, da der Nachweis einer Organdysfunktion für die Diagnose einer Sepsis nun unabdingbar geworden ist. Folglich werden nur noch die Entitäten Sepsis und septischer Schock unterschieden ( $\bullet$ Abb. 1). Es sollte der Tatsache Rechnung getragen werden, dass pathophysiologisch eine Sepsis nicht nur mit einer generalisierten Entzündungsreaktion, sondern mit zirkulatorischen, zellulären und metabolischen Veränderungen einhergeht. Vor allem sollte eine Sepsis von einer unkomplizierten, nicht lebensbedrohlichen Infektion abgegrenzt werden. Insbesondere dem bei septischen Patienten veränderten zellulären Metabolismus wird eine wichtige Bedeutung beigemessen. Da die alleinige Fokussierung auf die immunologischen Prozesse in der Pathophysiologie der Sepsis verlassen wurde, wird auch der septische Schock jetzt neu definiert.

Merke

Der septische Schock ist eine Untergruppe in der Sepsis. In dieser sind die zugrunde liegenden zirkulatorischen, zellulären und metabolischen Veränderungen mit einem größeren Letalitätsrisiko assoziiert als bei einer alleinigen Sepsis. 
- Tab. 1 Sequential Organ Failure Assessment Score (SOFA-Score) [6, 7].

\begin{tabular}{|c|c|c|c|c|c|}
\hline SOFA-Score-Punkte & 0 & 1 & 2 & 3 & 4 \\
\hline $\begin{array}{l}\text { Lunge: } \mathrm{PaO}_{2} / \mathrm{FiO}_{2} \text {, } \\
\text { mmHg (Horovitz- } \\
\text { Quotient) }\end{array}$ & $\geq 400$ & $<400$ & $<300$ & $\begin{array}{l}<200 \text { mit maschinel- } \\
\text { ler Beatmung }\end{array}$ & $\begin{array}{l}<100 \text { mit maschi- } \\
\text { neller Beatmung }\end{array}$ \\
\hline $\begin{array}{l}\text { Gerinnung: Throm- } \\
\text { bozyten } \times 10^{3} / \mathrm{mm}^{3}\end{array}$ & $\geq 150$ & $<150$ & $<100$ & $<50$ & $<20$ \\
\hline $\begin{array}{l}\text { Leber: Bilirubin mg/dl } \\
(\mu \mathrm{mol} / \mathrm{I})\end{array}$ & $<1,2(<20)$ & $1,2-1,9(20-32)$ & $2,0-5,9(33-101)$ & $6,0-11,9(102-204)$ & $>12,0(>204)$ \\
\hline $\begin{array}{l}\text { Herz/Kreislauf: } \\
\text { Hypotension, arte- } \\
\text { rieller Mitteldruck }\end{array}$ & $\mathrm{MAD} \geq 70 \mathrm{mmHg}$ & $\mathrm{MAD}<70 \mathrm{mmHg}$ & $\begin{array}{l}\text { Dopamin }<5 \text { oder } \\
\text { Dobutamin (jede } \\
\text { Dosierung) }^{*}\end{array}$ & $\begin{array}{l}\text { Dopamin } 5,1-15 \text { oder } \\
\text { Adrenalin } \leq 0,1 \text { oder } \\
\text { Noradrenalin } \leq 0,1^{*}\end{array}$ & $\begin{array}{l}\text { Dopamin }>15 \text { oder } \\
\text { Adrenalin }>0,1 \text { oder } \\
\text { Noradrenalin }>0,1^{*}\end{array}$ \\
\hline $\begin{array}{l}\text { ZNS: Glasgow Coma } \\
\text { Scale }\end{array}$ & 15 & $13-14$ & $10-12$ & $6-9$ & $<6$ \\
\hline $\begin{array}{l}\text { Niere: Kreatinin mg/dl } \\
(\mu \mathrm{mol} / \mathrm{l}) \text { oder Diurese }\end{array}$ & $<1,2(<110)$ & $\begin{array}{l}1,2-1,9(110- \\
170)\end{array}$ & $\begin{array}{l}2,0-3,4(171- \\
299)\end{array}$ & $\begin{array}{l}3,5-4,9(300-400) \\
\text { oder }<500 \mathrm{ml} / \mathrm{d}\end{array}$ & $\begin{array}{l}>5,0(>440) \\
\text { oder }<200 \mathrm{ml} / \mathrm{d}\end{array}$ \\
\hline
\end{tabular}

\section{SOFA- und qSOFA-Score}

Die demnach für die Diagnosestellung der Sepsis obligate Organdysfunktion kann durch eine akute Änderung von mind. 2 Punkten im SOFA-Score (SOFA: Sequential Organ Failure Assessment, > Tab. 1) im Rahmen einer Infektion identifiziert werden $[6,7]$. Bei Patienten ohne vorbestehende Organdysfunktion soll ein SOFA-Score von 0 angenommen werden. Ein SOFA-Score von $\geq 2$ Punkten entspricht einem Mortalitätsrisiko von > 10\% [5].

Patienten mit septischem Schock können identifiziert werden

- durch das Vorliegen einer Sepsis,

- einhergehend mit persistierender Hypotension und der Notwendigkeit einer Vasopressorgabe (zur Aufrechterhaltung eines arteriellen Mitteldruckes von $\geq 65 \mathrm{mmHg}$ ) und

- einem Laktat von > 2 mmol/l trotz adäquater Volumengabe.

Der septische Schock ist demnach eine Form der Sepsis, bei der zugrunde liegende zirkulatorische und zellulärmetabolische Pathologien so stark ausgeprägt sind, dass das Mortalitätsrisiko zusätzlich signifikant erhöht ist (>40\%) [5].

Viele der im SOFA-Score enthaltenen klinischen Parameter werden außerhalb der Intensivstation routinemäßig nicht regelhaft bestimmt. Daher wurde im Rahmen der Sepsis-3-Definition ein neuer Score (quick-SOFA oder qSOFA) entwickelt. Dieser soll eine schnelle Risikoeinschätzung von Patienten in der Notaufnahme oder auf Normalstation ermöglichen, die ein erhöhtes Risiko für ein Organversagen haben und schnell identifiziert werden sollten ( $\triangleright$ Abb. 2).

\section{Merke}

Der qSOFA-Score nutzt zur Risikostratifizierung die Variablen veränderter mentaler Status, systolischer Blutdruck $\leq 100 \mathrm{mmHg}$ und Atemfrequenz von $\geq 22$ | $\min [5]$.

Besteht dann der Verdacht auf eine Sepsis, muss der SOFA-Score zum Einsatz kommen: Es wird überprüft, ob ein Anhalt für eine Organdysfunktion vorliegt, der Patient die neue Sepsis-3-Definition erfüllt und damit eine lebensbedrohliche Sepsis diagnostiziert werden kann. Falls dies zutrifft, muss im nächsten Schritt geklärt werden, ob der Patient die Kriterien für einen septischen Schock erfüllt: Vasopressoren trotz adäquater Volumengabe, um den mittleren arteriellen Druck $\geq 65 \mathrm{mmHg}$ zu halten und Laktat $>2 \mathrm{mmol} / \mathrm{I}$.

\section{Merke}

Laktatkonzentration und Vasopressorenbedarf haben unabhängig voneinander wesentlichen Einfluss auf die Überlebensrate und sind daher wesentlicher Bestandteil der neuen Definition des septischen Schocks.

Eine Hyperlaktatämie erhöht unabhängig vom Vasopressorbedarf das Letalitätsrisiko. So stieg die Letalität bei Patienten massiv an

- von schwerer Sepsis (Laktat <2,5 mmol/l, keine Vasopressoren, Letalität 14,2\%)

- über vasoplegischen Schock (Laktat <2,5 mmol/l, Vasopressoren ja, Letalität 27,7\%),

- kryptischen Schock (Laktat $\geq 2,5 \mathrm{mmol} / \mathrm{l}$, keine Vasopressoren, Letalität 35,3\%)

- bis zum dysoxischen Schock (Laktat $\geq 2,5 \mathrm{mmol} / \mathrm{l}$, Vasopressoren ja, Letalität 44,8\%) [8]. 
Bewusstseinsveränderung

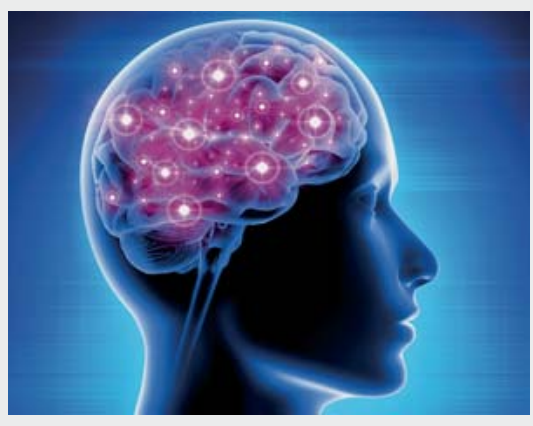

GCS $<15$
Tachypnoe

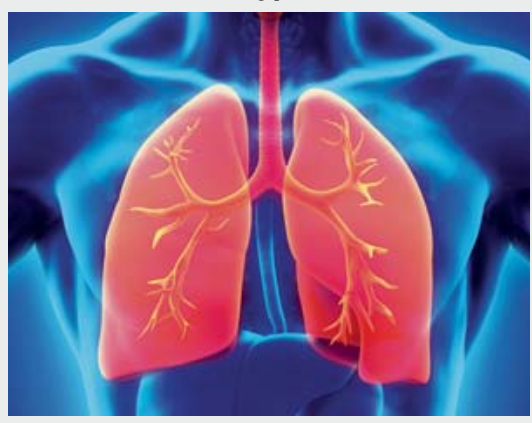

Atemfrequenz $\geq 22 / \mathrm{min}$
Hypotonie

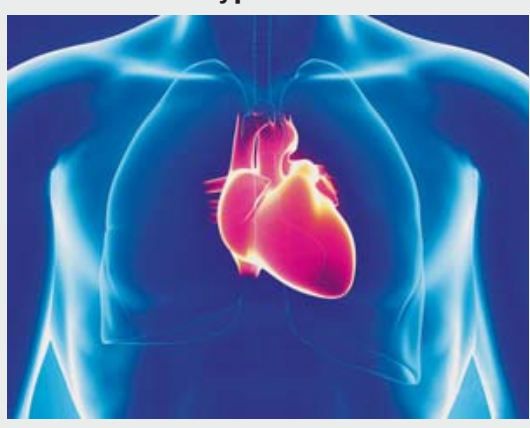

RRsys $\leq 100 \mathrm{mmHg}$

- Abb. 2 Kriterien des qSOFA-Scores. Der qSOFA ist ein evidenzbasierter klinischer Score, um vorwiegend außerhalb der Intensivstation Patienten mit erhöhtem Risiko für schwere Krankheitsverläufe zu identifizieren (erhöhte Wahrscheinlichkeit der Notwendigkeit einer Intensivtherapie und eine erhöhte Krankenhaussterblichkeit) [5]. GCS: Glasgow Coma Scale, RRsys: systolischer Blutdruck (Quelle: Gehirn: @ psdesign1 - stock.adobe. com; Lunge: @ nerthuz - stock.adobe.com; Herz: @ cliparea.com - stock.adobe.com).

\section{FALLBEISPIEL}

\section{Septischer Schock}

Eine 38-jährige Patientin ohne relevante Vorerkrankungen erhält elektiv eine laparoskopische Myomenukleation bei Uterus myomatosus. Bei der Visite am 2. postoperativen Tag fällt die Patientin mit einer plötzlichen Änderung der Bewusstseinslage auf. Während der anschließenden Kontrolle der Vitalparameter auf Normalstation zeigen sich zudem eine Hypotension (RRsys $<100 \mathrm{mmHg}$ ) sowie eine Tachypnoe. Das Abdomen der Patientin imponiert prall gespannt mit Abwehrspannung. Im Labor ist ein Anstieg der Leukozyten auf $17 \mathrm{G} / \mathrm{l}$ und des CRP auf $150 \mathrm{mg} / \mathrm{l}$ zu verzeichnen.

Nach Alarmierung des innerklinischen Notfallteams wird die Patientin mit der Verdachtsdiagnose einer abdominellen Sepsis auf die Intensivstation übernommen. Nach Abnahme von Urin- und Blutkulturen, dem sofortigen Beginn einer breiten Antibiose und der hämodynamischen Stabilisierung zeigt sich im CT eine Hohlorganperforation mit freier Luft. Daher wird die Indikation zur sofortigen explorativen Laparotomie gestellt. Trotz adäquater Volumengabe benötigt die Patientin im Verlauf Katecholamine, um einen arteriellen Mitteldruck von > 65 mmHg zu erreichen. Das Serum-Laktat liegt bei $2,5 \mathrm{mmol} / \mathrm{l}$. Aufgrund der Verwirrtheit, des Anstiegs des Serum-Kreatinins von Normwerten auf $150 \mu \mathrm{mol} / \mathrm{l}$ und der anhaltenden Katecholaminpflicht wird die Diagnose eines septischen Schocks gestellt. Die Patientin wird unverzüglich zur Fokussanierung in den OP verbracht.

\section{Kritik an Sepsis-3}

Die neue Sepsisdefinition wird sehr kontrovers diskutiert. Als Hauptargument gegen die neue Definition wird angeführt, dass diese den Therapiebeginn verzögern und damit die Prognose von septischen Patienten verschlechtern könnte. Dies könnte durch die Tatsache bedingt sein, dass für die Diagnosestellung der Sepsis bereits eine manifeste Organdysfunktion vorhanden sein muss (SOFA-Score $\geq 2$ ) - dies soll ja eigentlich durch einen frühzeitigen Therapiebeginn und optimales hämodynamisches Management gemäß der Surviving Sepsis Campaign Guidelines verhindert werden [9]. Darüber hinaus sind für die Ermittlung des SOFA-Scores Laborabnahmen notwendig, die einen hohen Zeitverlust mit sich bringen [10].

Weiterhin wird angeführt, dass bei der Entwicklung der Sepsis-3-Definition eine hohe Spezifität auf Kosten der Sensitivität in Kauf genommen wurde. In der täglichen klinischen Arbeit sind u. U. die Risiken einer Nichtbehandlung von falsch negativen Patienten als schwerwiegender einzustufen als die einer Überbehandlung von falsch positiven Patienten.

Schließlich ist unklar, inwiefern die Sepsis-3-Definition bei Patienten mit schwerwiegenden chronischen Organdysfunktionen (schwere COPD, terminale Niereninsuffizienz, Z.n. Apoplex oder Schädel-Hirn-Trauma, Leberzirrhose, Aplasie) angewendet werden kann - denn die Sepsis soll ja mithilfe eines Anstiegs des SOFA-Scores um 2 Punkte diagnostiziert werden [10].

Darüber hinaus wurden bei der Sepsis-3-Definition keine Biomarker zur Diagnosefindung mit aufgenommen (z. B. Procalcitonin oder Interleukin-6), obwohl diese weltweit 
seit vielen Jahren in der Routinediagnostik verwendet werden.

\section{FALLBEISPIEL}

\section{Chronische Organdysfunktionen}

Ein 85-jähriger Patient wird vom Rettungsdienst mit AZ-Verschlechterung und seit 2 Wochen bestehenden Rückenschmerzen in die interdisziplinäre Notaufnahme gebracht. An Vorerkrankungen sind ein arterieller Hypertonus, eine COPD Stadium GOLD IV, eine chronische Niereninsuffizienz und ein Z. n. Apoplex vor 5 Jahren bekannt. Im Aufnahmelabor zeigen sich eine Leukozytose mit $18 \mathrm{G} / \mathrm{I}$ sowie ein mit 120 mg/l erhöhtes CRP. Darüber hinaus wird im Ohr eine Temperatur von $39,5^{\circ} \mathrm{C}$ gemessen. Der Patient zeigt bezüglich der zerebralen, kardiozirkulatorischen, pulmonalen und renalen Funktion keine Verschlechterung im Vergleich zum im letzten Arztbrief dokumentierten Ausgangsbefund. Urin- und Blutkulturen werden abgenommen. Das Abdomen imponiert klinisch unauffällig. Ein Röntgenthorax erbringt keine Hinweise auf Infiltrate. Im konventionellen Röntgen gibt es keine Hinweise auf Frakturen der Wirbelsäule. Nach Gabe von Perfalgan (Paracetamol) entfiebert der Patient und wird zur weiteren Behandlung auf die Normalstation verlegt.

Am Folgetag wird von der Mikrobiologie Staphylococcus aureus in allen Blutkulturen nachgewiesen. Eine Antibiose mit Flucloxacillin wird sofort begonnen. Dennoch entwickelt der Patient in den folgenden Stunden eine Hypotension mit Katecholaminpflicht trotz adäquater Volumengabe und ein Acute-on-chronic-Nierenversagen und wird daher zeitnah auf die Intensivstation verlegt. Als wahrscheinlichster Fokus wird im MRT eine Spondylodiszitis auf Höhe BWK 5/6 diagnostiziert. Trotz maximaler Intensivtherapie und operativer Fokussanierung entwickelt der Patient im weiteren Verlauf ein Multiorganversagen. Bei vorliegender Patientenverfügung wird die Therapie daher im Sinne einer Palliation modifiziert und der Patient verstirbt 72 h nach Aufnahme.

\section{Epidemiologie}

\section{Daten zur Sepsis}

Die neuen Definitionen erschweren auch die Interpretation epidemiologischer Daten zur Sepsis.

\footnotetext{
Merke

Je nach zugrunde gelegten Sepsisdefinitionen und Grenzwerten zur Organdysfunktion erhält man eine unterschiedliche Anzahl an Patienten mit Sepsis, Sepsis mit Organdysfunktionen bzw. mit septischem Schock sowie Letalitätsraten [11].
}

Dies ist wahrscheinlich noch bedeutender, wenn die Sepsis-3-Definition mit älteren Definitionen verglichen wird [10].
Die Punktprävalenz für Sepsis bzw. schwere Sepsis/septischer Schock nach alter Sepsisdefinition betrug 2001 auf deutschen Intensivstationen 12,4 bzw. 11,4\% [12]. Die Prävalenz der Sepsis wurde hierbei auf deutschen Intensivstationen auf 76-110/100000 erwachsene Einwohner in Deutschland hochgerechnet [12]. Die Intensivstationsbzw. Gesamtkrankenhausletalität lag bei schwerer Sepsis/septischem Schock bei 48,4 bzw. 55,2\%. Die Gesamtkrankenhausletalität lag bei schwerer Sepsis allein bei $47,3 \%$ und im septischen Schock bei 62,4\% [12].

\section{INSEP-Studie}

Die aktuellsten epidemiologischen Daten aus Deutschland wurden im Rahmen der INSEP-Studie 2013 an 11883 Patienten auf Intensivstationen erhoben [13]. Hierbei lag die Punktprävalenz nach alter Sepsisdefinition für schwere Sepsis/septischen Schock bei 17,9\% [13]. Erstmalig erfasst, betrug die Inzidenzrate für neu aufgetretene schwere Sepsis/septischen Schock 11,6/1000 Intensivstationstage [13].

Die Intensivstations- bzw. Gesamtkrankenhausletalität lag in der INSEP-Studie mit den alten Sepsisdefinitionen ohne Sepsis bei 6 bzw. 9,6\%, mit schwerer Sepsis bei 16,7 bzw. 23,4\% sowie im septischen Schock bei 37,3 bzw. $43,3 \%$. Wurde die neue Sepsis-3-Definition dem septischen Schock zugrunde gelegt, war jedoch die Intensivstations- bzw. Gesamtkrankenhausletalität deutlich höher bei 44,3 bzw. 50,9\% [13]. Nach Sepsis-1-Definition hatten 1285 Patienten einen septischen Schock, nach Sepsis-3-Definition 848 Patienten. Das bedeutet, dass beinahe ein Drittel der Patienten nicht mehr als Patienten im septischen Schock erfasst worden wären [13].

Die Punktprävalenz schwerer Sepsis war in dieser Studie im Vergleich zur deutschen Prävalenzstudie höher [10]. Diese unterschiedlichen Ergebnisse können teilweise durch unterschiedliche Definitionen von Organdysfunktionen in den beiden Studien bedingt sein - ein Problem, das epidemiologische Vergleiche erschwert. Nicht zuletzt durch die Einteilung der arteriellen Hypoxämie wurden in der INSEP-Studie mehr Patienten der schweren Sepsisgruppe zugeordnet als in der deutschen Prävalenzstudie $[12,13]$. Das Gegenteil gilt für die arterielle Hypotonie und die metabolische Azidose: Auf deren Basis wurden im Vergleich zur deutschen Prävalenzstudie weniger Patienten der INSEP-Studie als schwere Sepsis eingestuft.

\section{DRG-Statistik}

In Deutschland ist die Diagnose Sepsis abrechnungsrelevant. Eine Auswertung der deutschlandweiten fallpauschalenbezogenen Krankenhausstatistik (DRG-Statistik) von 2007 bis 2013 zeigte: Die Fallzahl der Sepsis anhand ICD-10-Codes für Sepsis stieg jährlich um 5,7\% von 200535 im Jahr 2007 auf 279530 Fälle 2013 an. Dies entsprach einer Zunahme der adjustierten Krankenhausrate an Sepsisfällen von 256/100000 auf 335/100000 Ein- 
wohner [14]. Der Anteil von Patienten mit schwerer Sepsis nahm von 27 auf $41 \%$ zu, wobei 2013 12\% einen septischen Schock aufwiesen [14]. Die Sterblichkeitsrate der Sepsis sank im Beobachtungszeitraum um 2,7 auf 24,3\% [13]. 2013 starben damit 67849 Menschen an oder mit einer Sepsis [14]. Die Fallzahlraten waren in den extremen Altersgruppen am höchsten und die Krankenhausletalität nahm ab dem 40. Lebensjahr nahezu linear zu [14].

Die kodierten Fälle mit septischem Schock stiegen von 2010 bis 2013 von 22326 auf 33815 an, wobei die Krankenhaussterblichkeit von 61,0 auf $58,8 \%$ abfiel [14]. Das Bundesversicherungsamt erhob 2013 mittlere Leistungsausgaben von 27467,92 Euro pro Sepsisfall. Hieraus errechneten sich für 2013 Gesamtkosten von rund 7,7 Milliarden Euro für die stationäre und nachfolgende ambulante Behandlung der Sepsisfolgen in Deutschland [14].

\section{Daten zu Infektionen auf Intensivstationen}

\section{Erreger und Infektionen}

Die EPIC-II-Studie wies in Europa 2007 bei 62\% der Patienten gramnegative Erreger, bei $47 \%$ grampositive Erreger sowie bei $19 \%$ Pilze nach [15]. Als häufigste Erreger wurden detektiert:

- im gramnegativen Bereich Escherichia coli, Pseudomonas aeruginosa, Klebsiellae sowie

- im grampositiven Bereich Staphylococcus aureus, Staphylococcus epidermidis, Streptococcus pneumoniae [15].

S.-aureus-Nachweis in Blutkulturen bedeutete fast immer S.-aureus-Blutstrominfektion (Staphylococcus aureus Bloodstream Infection = SABSI) und praktisch nie eine Kontamination. Dies war verbunden mit einem hohen Risiko für Endokarditis [16] in 10-15\%, einem häufigeren Nachweis von Methicillin-resistenten Stämmen (MRSA) und einer Inzidenz von ca. 15-35/100 000/Jahr. Häufigste Eintrittspforten waren infizierte intravaskuläre Katheter (zentrale Venenkatheter, Ports, periphere Venenkatheter) sowie der Respirationstrakt.

In der EPIC-II-Studie hatten 7087 von 13796 erwachsenen Patienten von 1265 Intensivstationen aus 75 Ländern Infektionen [17]. Die häufigsten Infektionen betrafen den Respirationstrakt (63\%), gefolgt von abdominellen Infektionen (20\%), Blutstrominfektionen (15\%) und Harnwegsinfekten (14\%). Die mikrobiologischen Kulturen waren bei $67 \%$ dieser Patienten positiv, meist mit gramnegativen Bakterien (48,0\%); Antibiotika wurden bei $98,1 \%$ dieser Patienten verabreicht [17]. Die Intensivstationsbzw. Gesamtkrankenhausletalität lag bei 29,4 bzw. $36,3 \%$, wobei sie bei Patienten mit abdominellen Infektionen höher war als bei Patienten mit anderen Infektionen (29,4 vs. $24,4 \%$ [ [17]. In einer multivariaten Analyse waren hämatologische Erkrankungen, maschinelle Beat- mung, Zirrhose, Nierenersatzverfahren sowie ein hoher Schweregrad der Erkrankung (SAPS-II-Score) unabhängige Risikofaktoren für eine erhöhte Letalität [17].

INFO

Aktuelle deutsche Daten

Die aktuellsten Daten aus Deutschland zeigten in der MEDUSA-Studie [18] 2011-2013 (Interventionsgruppe/Kontrollgruppe) eine Verteilung des Fokus der Infektion in fallender Häufigkeit: Pneumonie $(35,4 / 37,7 \%)$, intraabdominelle Infektionen $(35,7)$ $35,8 \%)$, urogenitale Infektionen $(12,1 / 13,6 \%)$ und primäre Bakteriämien (1,6/1,5\%). In der INSEP-Studie 2013 [13] zeigte sich folgende Fokusverteilung: Pneumonie 46,6\%, intraabdominelle Infektionen $28,7 \%$, urogenitale Infektionen $12,6 \%$ und primäre Bakteriämien 2,2\%. Die Rate positiver Blutkulturen ist mit 20-40\% niedrig, so waren jüngst von 4858 abgenommenen Blutkulturen 59\% negativ bzw. kontaminiert und lediglich $41 \%$ positiv [19].

\section{Fulminante Verlaufsformen}

Eine besonders schlechte Prognose haben fulminante Verlaufsformen der Sepsis, wie

- Meningokokkensepsis (petechiale Hautblutungen und Verbrauchskoagulopathie, evtl. mit bilateraler Nebennierennekrose „Waterhouse-Friderichsen-Syndrom“)

- Sepsis nach Splenektomie (Overwhelming Post-Splenectomy Infection = OPSI) [20]

- S.-aureus-Blutstrominfektion (SABSI) mit hohem Risiko für Endokarditis [16]. Daher ist eine wiederholte Abnahme von Blutkulturen und 2-wöchige Antibiotikatherapie nach der ersten negativen Blutkultur notwendig, um die Ausbildung einer Endokarditis zu erkennen und ggf. zu vermeiden. Zudem ist eine Bildgebung mittels transösophagealer Echokardiografie (TEE) bei allen SABSI-Patienten zum Ausschluss einer Endokarditis erforderlich. Eine alleinige transthorakale Echokardiografie (TTE) ist nur bei unkomplizierten SABSI-Fällen nach strenger Definition sowie bei Kontraindikationen zur TEE zu erwägen - denn in bis zu $20 \%$ kommt es zu falsch negativen TTE-Befunden.

- Landouzy-Sepsis: septische Verlaufsform der Tuberkulose bei Immunsuppression

- Toxic Shock Syndrome (TSS)

- staphylokokkenassoziiertes TSS durch TSS-Toxin 1 bei vaginalen Infektionen, z. B. „tamponassoziiertes Schocksyndrom“ (Bildung von Exotoxin C, Enterotoxin $\mathrm{F}$ )

- streptokokkenassoziiertes TSS durch Enterotoxine der Gruppe-A-Streptokokken (GAS) bei nekrotisierender Fasziitis oder Myositis 


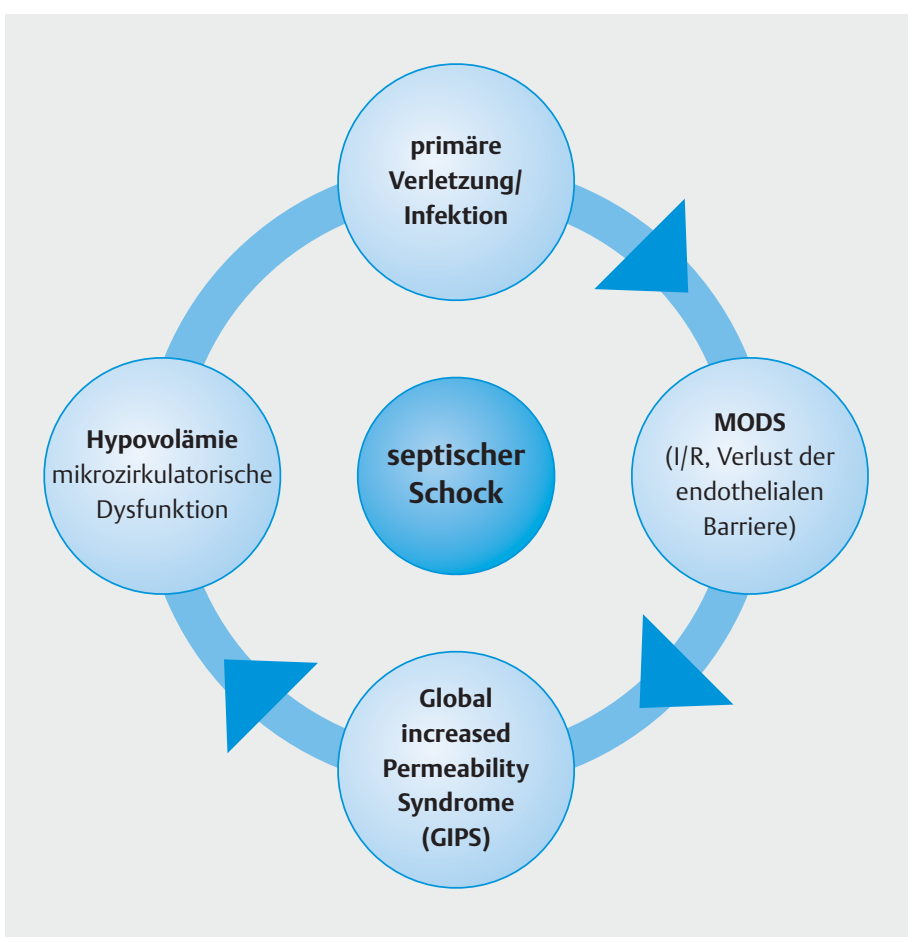

- Abb. 3 „Four Hit Model“ der Sepsis. MODS: Multiple Organ Dysfunction Syndrome, I/R: Ischämie/Reperfusion.

\section{Pathophysiologie}

Pathophysiologisch können mehrere Phasen der Organschädigungen unterschieden werden (s. „Info - Four Hit Model“) [21]. Als entscheidender Wendepunkt wird der 3. Tag nach Schockbeginn angesehen. Dann folgt bei günstigem Verlauf auf den initialen distributiven Schock (arterielle Vasodilatation und transkapillärer Albuminverlust) die hämodynamische Stabilisierung: Es kommt zum „Verschließen“ des kapillären Lecks, Wiederherstellung der Diurese und Mobilisation von extravaskulärer Flüssigkeit [21].

Cave

Eine zu starke Positivbilanzierung von Sepsispatienten ist mit einem negativen Ausgang assoziiert.

So zeigte sich bei Sepsispatienten, die am ersten Tag 5 bis >9 I Flüssigkeit erhielten: Mit jedem zusätzlichen Liter über 5 I stiegen die Letalität um 2,3\% sowie die Krankenhauskosten um $999 \$[22]$.

Differenzialdiagnostisch abzugrenzen sind hypovolämische, kardiogene, neurogene und anaphylaktische Schockformen.
INFO

„Four Hit Model“

Das „Four Hit Model“ der Organschädigungen bei

Sepsis differenziert

- „first hit“ = akute Verletzung/akuter Infekt (z. B. Pneumonie),

- "second hit“ = Multiorgandysfunktionssyndrom (z. B. Ischämie-Reperfusion, toxische Sauerstoffmetabolite, Verlust kapillärer Endothel-Barrierefunktion),

- "third hit“ = Global increased Permeability Syndrome (GIPS) und

- „fourth hit“ = negative kumulative Flüssigkeitsbilanz und resultierende Hypovolämie ( $\triangleright$ Abb. 3).

\section{Prognose}

Patienten mit einer Sepsis sind sehr heterogen bezüglich

- der Quelle der Infektion und der auslösenden Mikroorganismen (Menge und Virulenz),

- genetischem Hintergrund,

- Alter und Komorbiditäten,

- chronischer Medikation und

- Lebensführung (PIRO-Konzept: Prädisposition, Infektion, Patientenantwort auf Infektion [Response], Organdysfunktionen).

Daraus resultiert eine hohe Variabilität bezüglich Immunantwort, Überlebensrate und Nutzen potenzieller Therapien.

Von den geschätzten jährlich 19 Mio. Patienten mit einer Sepsis überleben ca. 14 Mio. [23]. Die Hälfte der Überlebenden erholt sich, ein Drittel verstirbt während des folgenden Jahres und ein Sechstel behält andauernde Beeinträchtigungen [23]. Diese Beeinträchtigungen umfassen

- im Schnitt 1-2 funktionelle Einschränkungen,

- einen 3-fachen Anstieg der Prävalenz milder bis schwerer kognitiver Beeinträchtigungen und

- eine hohe Prävalenz psychischer Gesundheitsprobleme wie Angst (32\% der Überlebenden), Depressionen (29\%) oder posttraumatische Belastungsstörungen (44\%) [23].

Ungefähr 40\% der Patienten müssen nach Krankenhausentlassung innerhalb von 90 Tagen wieder aufgenommen werden [23]. Im Vergleich zu anderen hospitalisierten Patienten haben Überlebende einer Sepsis ein erhöhtes Infektionsrisiko (8\%), eine erhöhte Rate akuten Nierenversagens (3,3 vs. $1,1 \%$ ) und neuer kardiovaskulärer Ereignisse (Hazard Ratio 1,1-1,4) [23]. Die Ursachen der Verschlechterung sind multifaktoriell und beinhalten eine beschleunigte Progression vorbestehender chronischer Erkrankungen, verbleibende Organschädigungen und eingeschränkte Immunfunktion [23]. Dies unterstreicht die Notwendigkeit 
- poststationärer Rehabilitation,

- der Identifikation psychischer und kognitiver Probleme,

- der Anpassung einer Langzeitmedikation,

- einer engmaschigen ambulanten Therapie sowie

- ggf. der Einleitung einer palliativen Therapie bei schlechtem bzw. abbauendem Gesundheitsstatus [23].

Hierbei muss auch an die Angehörigen gedacht werden: Bis zu 69\% der Überlebenden einer Sepsis und 62\% der Ehepartner litten an klinischen Zeichen einer posttraumatischen Belastungsstörung [24]. Sepsis ist ein wesentlicher Risikofaktor für die bei $25-100 \%$ der Patienten berichtete Inzidenz von auf der Intensivstation erworbener Muskelschwäche [25].

\section{Merke}

Schwere Sepsis und septischer Schock sind die dritthäufigste Todesursache im Krankenhaus! Jeder Zeitverlust vor Beginn einer effektiven Therapie verschlechtert die Prognose!

\section{KERNAUSSAGEN}

- Eine Definition des Krankheitsbildes der Sepsis mit hoher diagnostischer Sensitivität und Spezifität ist entscheidend für eine frühzeitige Behandlung der Patienten mit Sepsis.

- Als Sepsis wird eine lebensbedrohliche Organdysfunktion definiert, die durch eine dysregulierte Immunantwort des Patienten auf eine Infektion verursacht wird.

- Bei Patienten im septischen Schock sind die zugrunde liegenden zirkulatorischen, zellulären und metabolischen Veränderungen mit einem größeren Letalitätsrisiko assoziiert als bei Patienten mit einer alleinigen Sepsis.

- Die Intensivstations- bzw. Gesamtkrankenhausletalität lag in der INSEP-Studie mit den alten Sepsisdefinitionen im septischen Schock bei 37,3 bzw. 43,3\%. Mit der neuen Sepsis-3-Definition betrug sie 44,3 bzw. 50,9\%.

- Schwere Sepsis und septischer Schock sind die dritthäufigste Todesursache im Krankenhaus.

\section{Interessenkonflikt}

Die Autoren geben an, dass kein Interessenkonflikt besteht.

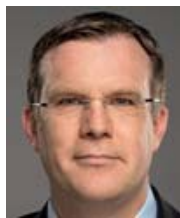

\section{Hendrik Brach}

Prof. Dr. med., Jahrgang 1973. Studium der Humanmedizin in Ulm und Bern. 2006-2011 Facharztausbildung Anästhesie Universitätsklinikum Ulm, Zusatzbezeichnungen Notfallmedizin und spezielle Intensivmedizin. Seit 2011 Oberarzt, seit 2012 geschäftsführender Oberarzt der Klinik für Anästhesiologie Ulm. Schriftführer des wissenschaftl. Arbeitskreises Intensivmedizin DGAI, Forschungsgruppenleiter TIFOnet, ABS-Experte (DGKH).

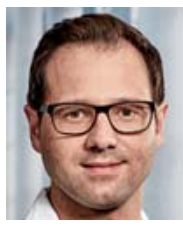

\section{Sebastian Hafner}

PD Dr. med., Jahrgang 1982. Studium der Humanmedizin in Ulm und Bern. 2008-2013 Facharztausbildung Anästhesie am Universitätsklinikum Ulm und am Bundeswehrkrankenhaus Ulm, Zusatzbezeichnungen Notfallmedizin und spezielle Intensivmedizin. Seit 2015 Oberarzt der Klinik für Anästhesiologie am Universitätsklinikum Ulm. 2018 Habilitation für das Fach Anästhesiologie. Schwerpunkte: Operative Intensivmedizin, Organprotektion/-ersatz.

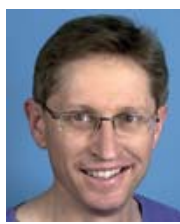

\section{Manfred Weiß}

apl. Prof. Dr. med., MBA, Jahrgang 1958. Studium der Humanmedizin an der Ruhr-Universität Bochum. 1984-1986, 1988-1995 Facharztausbildung Anästhesie Heinrich-Heine-Universität Düsseldorf. 1986-1988 Internistische Weiterbildung Universität Aachen. Seit 1995 Oberarzt der Klinik für Anästhesiologie des Universitätsklinikums Ulm. Schwerpunkte: Operative Intensivmedizin, Individualisierte Immundiagnostik/-therapie, Ethik, Personalbedarf.

Hendrik Bracht und Sebastian Hafner haben gleichermaßen zur Arbeit beigetragen.

\section{Korrespondenzadresse}

Prof. Dr. med. Hendrik Bracht

Klinik für Anästhesiologie

Universitätsklinikum Ulm

Albert-Einstein-Allee 23

89081 Ulm

hendrik.bracht@uniklinik-ulm.de

Wissenschaftlich verantwortlich gemäß Zertifizierungsbestimmungen

Wissenschaftlich verantwortlich gemäß Zertifizierungsbestimmungen für diesen Beitrag ist Prof. Dr. med. Hendrik Bracht, Ulm.

\section{Literatur}

[1] [Anonym]. American College of Chest Physicians/Society of Critical Care Medicine Consensus Conference: Definitions for sepsis and organ failure and guidelines for the use of innovative therapies in sepsis. Crit Care Med 1992; 20: 864-874 
[2] Levy MM, Fink MP, Marshall JC et al. 2001 SCCM/ESICM/ACCP/ ATS/SIS International Sepsis Definitions Conference. Intensive Care Med 2003; 29: 530-538

[3] Seymour CW, Liu VX, Iwashyna T] et al. Assessment of Clinical Criteria for Sepsis: For the Third International Consensus Definitions for Sepsis and Septic Shock (Sepsis-3). JAMA 2016; 315: 762-774. doi:10.1001/jama.2016.0288

[4] Shankar-Hari M, Phillips GS, Levy ML et al. Developing a New Definition and Assessing New Clinical Criteria for Septic Shock: For the Third International Consensus Definitions for Sepsis and Septic Shock (Sepsis-3). JAMA 2016; 315: 775-787. doi:10.1001/jama.2016.0289

[5] Singer M, Deutschman CS, Seymour CW et al. The Third International Consensus Definitions for Sepsis and Septic Shock (Sepsis-3). JAMA 2016; 315: 801-810. doi:10.1001/jama.2016.0287

[6] Vincent JL, Moreno R, Takala J et al. The SOFA (Sepsis-related Organ Failure Assessment) score to describe organ dysfunction/failure. On behalf of the Working Group on Sepsis-Related Problems of the European Society of Intensive Care Medicine. Intensive Care Med 1996; 22: 707-710

[7] Vincent JL, de Mendonça A, Cantraine F et al. Use of the SOFA score to assess the incidence of organ dysfunction/failure in intensive care units: results of a multicenter, prospective study. Working group on "sepsis-related problems" of the European Society of Intensive Care Medicine. Crit Care Med 1998; 26: 1793-1800

[8] Thomas-Rueddel DO, Poidinger B, Weiss $\mathrm{M}$ et al. Hyperlactatemia is an independent predictor of mortality and denotes distinct subtypes of severe sepsis and septic shock. J Crit Care 2015; 30: 439.e1-439.e6. doi:10.1016/j.jcrc.2014.10.027

[9] Rhodes A, Evans LE, Alhazzani W et al. Surviving Sepsis Campaign: International Guidelines for Management of Sepsis and Septic Shock: 2016. Intensive Care Med 2017; 43: 304-377. doi:10.1007/s00134-017-4683-6

[10] Marx G, Weigand MA. Sepsis-3: Zweifel an der Überlegenheit der neuen Definition. Dtsch Arztebl 2017; 114: A-1429/B$1201 / \mathrm{C}-1175$

[11] Weiss M, Huber-Lang M, Taenzer $M$ et al. Different patient case mix by applying the 2003 SCCM/ESICM/ACCP/ATS/SIS sepsis definitions instead of the 1992 ACCP/SCCM sepsis definitions in surgical patients: a retrospective observational study. BMC Med Inform Decis Mak 2009; 9: 25. doi:10.1186/ 1472-6947-9-25

[12] Engel C, Brunkhorst FM, Bone HG et al. Epidemiology of sepsis in Germany: results from a national prospective multicenter study. Intensive Care Med 2007; 33: 606-618. doi:10.1007/ s00134-006-0517-7

[13] SepNet Critical Care Trials Group. Incidence of severe sepsis and septic shock in German intensive care units: the prospective, multicentre INSEP study. Intensive Care Med 2016; 42: 1980-1989. doi:10.1007/s00134-016-4504-3
[14] Fleischmann C, Thomas-Rueddel DO, Hartmann M et al. Hospital Incidence and Mortality Rates of Sepsis. Dtsch Arztebl Int 2016; 113: 159-166. doi:10.3238/arztebl.2016.0159

[15] Vincent JL, Rello J, Marshall J et al. International study of the prevalence and outcomes of infection in intensive care units. JAMA 2009; 302: 2323-2329

[16] Le Moing V, Alla F, Doco-Lecompte T et al. Staphylococcus aureus bloodstream infection and endocarditis-a prospective cohort study. PloS One 2015; 10: e0127385. doi:10.1371/journal.pone. 0127385

[17] De Waele J, Lipman J, Sakr Y et al. Abdominal infections in the intensive care unit: characteristics, treatment and determinants of outcome. BMC Infect Dis 2014; 14: 420. doi:10.1186/1471-2334-14-420

[18] Bloos F, Ruddel H, Thomas-Ruddel D et al. Effect of a multifaceted educational intervention for anti-infectious measures on sepsis mortality: a cluster randomized trial. Intensive Care Med 2017; 43: 1602-1612. doi:10.1007/s00134-017-4782-4

[19] Thomas-Rüddel D, Poidinger B, Kott M et al.; MEDUSA study group. Influence of pathogen and focus of infection on procalcitonin values in patients with bacteremia or candidemia. Crit Care 2018; 22: 128. doi:10.1186/s13054-018-2050-9

[20] Edgren G, Almqvist R, Hartman M et al. Splenectomy and the risk of sepsis: a population-based cohort study. Ann Surg 2014; 260: 1081-1087. doi:10.1097/SLA.0000000000000439

[21] Malbrain ML, Marik PE, Witters I et al. Fluid overload, de-resuscitation, and outcomes in critically ill or injured patients: a systematic review with suggestions for clinical practice. Anaesthesiol Intensive Ther 2014; 46: 361-380. doi:10.5603/ AIT.2014.0060

[22] Marik PE, Linde-Zwirble WT, Bittner EA et al. Fluid administration in severe sepsis and septic shock, patterns and outcomes: an analysis of a large national database. Intensive Care Med 2017; 43: 625-632. doi:10.1007/s00134-016-4675-y

[23] Prescott HC, Angus DC. Enhancing recovery from sepsis: a review. JAMA 2018; 319: 62-75. doi:10.1001/jama.2017.17687

[24] Rosendahl J, Brunkhorst FM, Jaenichen D et al. Physical and mental health in patients and spouses after intensive care of severe sepsis: a dyadic perspective on long-term sequelae testing the Actor-Partner Interdependence Model. Crit Care Med 2013; 41: 69-75. doi:10.1097/CCM.0b013e31826766b0

[25] Kress JP, Hall JB. ICU-acquired weakness and recovery from critical illness. N Engl J Med 2014; 370: 1626-1635. doi:10.1056/NEJMra1209390

Bibliografie

DOI https://doi.org/10.1055/a-0625-5492

Anästhesiol Intensivmed Notfallmed Schmerzther 2019; 54: 10-20 @ Georg Thieme Verlag KG Stuttgart · New York ISSN 0939-2661 


\section{Punkte sammeln auf CME.thieme.de}

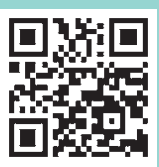

Diese Fortbildungseinheit ist 12 Monate online für die Teilnahme verfügbar.

Sollten Sie Fragen zur Online-Teilnahme haben, finden Sie unter cme.thieme.de/hilfe eine ausführliche Anleitung. Wir wünschen viel Erfolg beim Beantworten der Fragen!

Unter eref/thieme.de/CXAY7D6 oder über den QR-Code kommen Sie direkt zum Artikel zur Eingabe der Antworten.

VNR 2760512019156641167

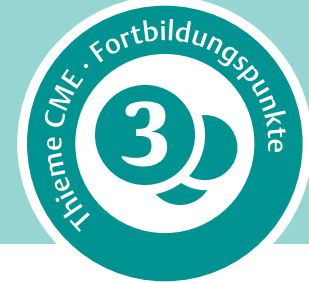

\section{Frage 1}

Welches der folgenden Kriterien gehört nicht zur Definition der Sepsis nach Sepsis-1?
A Linksverschiebung $>10 \%$
B Tachypnoe ( $\geq 20 / \mathrm{min})$
C flüssigkeitsrefraktäre Hypotonie mit konsekutiver Katechol- aminpflichtigkeit
D Hypothermie $\left(\leq 36^{\circ} \mathrm{C}\right)$
E vermutete Infektion

\section{Frage 2}

Welches Kriterium ist nicht Bestandteil des sog. qSOFA-Scores?
A neu aufgetretene Bewusstseinsveränderung
B Tachykardie
C Hypotension
D Tachypnoe
E GCS $<15$

\section{Frage 3}

Wie groß ist die erwartete Letalität bei einem septischen Schock nach der Sepsis-3-Definition?
A $5-10 \%$
B $20-30 \%$
C $35 \%$
D $>40 \%$
E $>60 \%$

\section{Frage 4}

Welche Aussage ist richtig? In der EPIC-II-Studie wurden die Häufigkeiten von Infektionen auf Intensivstationen untersucht. Die häufigsten Infektionen in dieser Studie waren...
A Blutstrominfektionen.
B Harnwegsinfekte.
C Haut- und Weichteilinfekt.
D Infektionen des Respirationstraktes.
E abdominelle Infektionen.

\section{Frage 5}

In der INSEP-Studie wurde erstmals die Inzidenzrate einer neu aufgetretenen schweren Sepsis berechnet? Wie hoch war diese?
$A \approx 5 / 1000$ Intensivstationstage
B $\approx 11 / 1000$ Intensivstationstage
$C \approx 16 / 1000$ Intensivstationstage
D $\approx 20 / 1000$ Intensivstationstage
$\mathrm{E} \approx 25 / 1000$ Intensivstationstage

\section{Frage 6}

Welche Aussage ist falsch?

A Nachweise von S. aureus in Blutkulturen sind praktisch nie Kontaminationen.

B Nachweise von S. aureus sind mit einem hohen Endokarditisrisiko verbunden.

C Nachweise von S. aureus und von Koagulase-negativen Staphylokokken sind gleich zu bewerten.

D Häufige Eintrittspforten sind intravasale Katheter und der Respirationstrakt.

E Die Inzidenz einer S.-aureus-Butstrominfektion beträgt ca. 15-35/100000/Jahr.

\section{- Weitere Fragen auf der folgenden Seite...}




\section{Punkte sammeln auf CME.thieme.de}

Fortsetzung $\ldots$

\section{Frage 7}

Wann kann gemäß der Sepsis-3-Definition ein septischer Schock diagnostiziert werden?

A beim Vorliegen einer Sepsis sowie gleichzeitig der Notwendigkeit einer Vasopressorgabe (zur Aufrechterhaltung eines arteriellen Mitteldruckes von $\geq 65 \mathrm{mmHg}$ ) trotz adäquater Volumengabe

B beim Vorliegen einer Sepsis sowie gleichzeitig der Notwendigkeit einer Vasopressorgabe (zur Aufrechterhaltung eines arteriellen Mitteldruckes von $\geq 65 \mathrm{mmHg}$ ) und einem Laktat von $>2 \mathrm{mmol} / \mathrm{l}$ trotz adäquater Volumengabe

$C$ beim Vorliegen einer Sepsis sowie gleichzeitig der Notwendigkeit einer Vasopressorgabe (zur Aufrechterhaltung eines arteriellen Mitteldruckes von $\geq 65 \mathrm{mmHg}$ ) und einem Laktat von > 1,5 mmol/l trotz adäquater Volumengabe

$D$ bei gleichzeitigem Vorliegen einer Infektion sowie einer Hypotension und einem Laktatanstieg von $>2 \mathrm{mmol}$

E beim Vorliegen einer Infektion sowie einer verminderten $\mathrm{DO}_{2}$ und gleichzeitig erniedrigter zentralvenöser Sättigung $\mathrm{ScvO}_{2}$

\section{Frage 8}

Wann liegt gemäß der Sepsis-3-Definition eine Sepsis vor?

A beim Auftreten von plötzlicher Verwirrtheit, Tachypnoe und einer Hypotension

B beim Auftreten eines Organversagens im Verlauf einer Infektion, das durch einen zu niedrigen arteriellen Mitteldruck ausgelöst wurde

$C$ bei eitriger Sekretion aus offenen Wunden und gleichzeitig Erfüllung von 2 Kriterien des qSOFA-Scores

$D$ bei Auftreten einer lebensbedrohlichen Organdysfunktion (Änderung SOFA-Score $\geq 2$ ), die durch eine dysregulierte Immunantwort des Patienten auf eine Infektion ausgelöst wird

$\mathrm{E}$ bei Nachweis von 2 der 4 SIRS-Kriterien und einer vermuteten oder nachgewiesenen Infektion

\section{Frage 9}

Welcher Parameter gehört nicht zu den pathogenetischen Faktoren im „Four Hit Model“ der Organschädigungen?

A negative kumulative Flüssigkeitsbilanz und resultierende Hypovolämie

B Euvolämie

C Global increased Permeability Syndrome (GIPS)

D akute Verletzung/akuter Infekt

E Multiorgandysfunktionssyndrom

\section{Frage 10}

Mit welcher Beeinträchtigung muss bei Patienten nach einer Sepsis nicht gerechnet werden?
A kognitive Beeinträchtigungen
B Adipositas
C Angst
D Depressionen
E Muskelschwäche 